\title{
Using simplified geometric models in skill-based manipulation for objects used in daily life
}

\author{
Akira Nakamura*1, Kazuyuki Nagata ${ }^{1}$, Kensuke Harada $^{2}$, Natsuki Yamanobe ${ }^{1}$ \\ ${ }^{1}$ Manipulation Research Group, Intelligent Systems Research Institute, National Institute of Advanced Industrial Science and \\ Technology (AIST), Ibaraki, Japan \\ ${ }^{2}$ Department of Systems Innovation, Graduate School of Engineering Science, Osaka University, Osaka, Japan
}

Received: June 18, 2017

DOI: $10.5430 /$ air.v6n2p100
Accepted: July 31, 2017

Online Published: August 10, 2017

\begin{abstract}
Recently many types of robots have been playing an active part in various fields. The operation of these robots' manipulators is an important subject of research. The tasks of manipulation can be regarded as sequences of several motion primitives called "skills". Skills also have ability to compensate for errors both in modeling and in execution. Data may occur in the elements of the shapes, positions and orientations of objects that can be dispensed with to make geometric models simpler. In order to achieve tasks with high reliability, this paper proposes simplified geometric models based on skill techniques not only for industrial products but also for objects used in the daily life of humans. As examples of simplified geometric models of objects used in daily life, simplified models in a transfer task of plastic bottles and in a transfer task of a cup are explained.
\end{abstract}

Key Words: Manipulation skill, Motion primitive, Geometric model, Planning, Visual sensing

\section{INTRODUCTION}

In recent years many robots have been playing an active part in various fields such as factories, gardens, as well as in the daily living environments of humans. For manipulation robots to be useful in many fields, it is necessary for them to be able to achieve a lot of works without failure by use of specialized methods. By investigating human behaviors in actual typical tasks, such as grasping, assembling, packing and transferring, we detected that the behaviors were composed of various important motion primitives. We call these primitives "skills" and have explained that many works of manipulators can be derived by sequences of skills. ${ }^{[1-4]}$ Although there are very many kinds of manipulation skills, the skills in which changes of the states of touch happen in the motion primitives that comprise the task performed are particularly important. The three motion primitives of "move-to-touch", "rotate-to-level" and "rotate-to-insert" are important skills in which the contact states vary. ${ }^{[1-7]}$ Most manipulation tasks in assembling and packing can be composed of these principal skills.

It is very important that the manipulation skills are also useful for error compensation both in visual sensing and model matching and in task planning. For example, modeling errors in the directions of transition can be cancelled in the moveto-touch skill. Similarly, model errors in the directions of rotation can be reduced in the rotate-to-level skill. Moreover, geometric model simplification can be derived by taking into account a maximum error or margin in the motions of the skill primitives. ${ }^{[8,9]}$ In skill-based manipulation, the model-

\footnotetext{
*Correspondence: Akira Nakamura; Email: a-nakamura@aist.go.jp; Address: Manipulation Research Group, Intelligent Systems Research Institute, National Institute of Advanced Industrial Science and Technology (AIST), Central 1, Umezono 1-1-1, Tsukuba, Ibaraki 305-8560, Japan.
} 
ing of an object can have margins for the shape, position and orientation. Therefore, models can be constructed simply using only the necessary data in model matching and motion planning. Since less data on the objects is needed for the shapes, positions and orientations, sensing and planning are easier.

Manipulation systems in our method are derived based on skill primitives composed of tasks. Similar techniques based on components have been reported as a discrete-event system. ${ }^{[10-12]}$ However, the simplification of the geometric models was not taken into account in those studies. Furthermore, a large number of studies on object modeling for manipulation have been undertaken recently. ${ }^{[13,14]}$ For example, there are studies on object modeling for grasping, object modeling that takes into account environmental knowledge, and object modeling for assemblies. ${ }^{[15-17]}$ However, there are no studies which deal at the same time with object modeling for both planning and sensing in manipulation. ${ }^{[18]}$

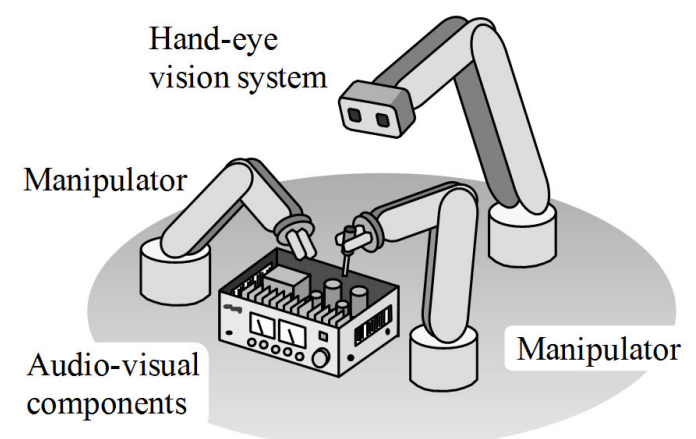

Figure 1. Manipulators of industrial robots working in factories

Simplified geometric models have been derived for industrial products in factories $^{[8,9]}$ (see Figure 1). In these cases, the data for the models were obtained precisely. In recent years, robots have begun to be active in human environments (see Figure 2). However, precise models for objects used in the daily life of humans are not always available in computer software. Therefore, a process composed of sensing, modeling, planning and execution is performed based on some inaccurate models. However, there is the possibility of canceling some model errors when using skill techniques like those mentioned above. In other words, when also using skill techniques for operations involving objects used in daily life, it becomes possible to perform manipulation with confidence that the operation will be achieved successfully. In this paper, in order to achieve tasks with high reliability, we propose simplified geometric models based on skill techniques for objects used in daily life.

Published by Sciedu Press
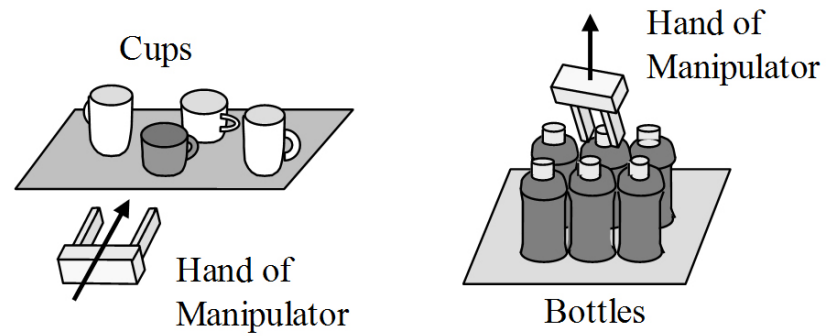

Figure 2. Manipulators of robots working in daily life

Manipulation skills are explained in the next section. The definition of a skill is given, and skill sequences are explained based on the process flow composed of sensing, modeling, planning, execution and so on. We show that skill techniques bring about model simplification in sensing and planning. A simplified model applied for an industrial product is shown in Section 4. A simplified model based on skills for an object used in daily life is proposed in Section 5.

\section{MANIPULATION SKILLS AND CONSTRUC- TION OF TASKS}

In this section, we explain our concept of skills in working robots and the processes to perform tasks composed of manipulation skills. A detailed explanation is available in References. $^{[1-4]}$

\subsection{Skill primitives}

In manipulation tasks such as grasping, assembling, packing and removing, there are skill primitives in which the states of contact vary. These skills are particularly important. In References, ${ }^{[5-7]}$ we considered the motion primitives of "move-to-touch", "rotate-to-level" and "rotate-to-insert", as principal skills, and many assembly tasks can be composed of these skills. After this, to simplify the explanation, we assume that the shapes of the grasped objects, the other objects and the holes are rectangular parallelepipeds, and view these skills as occurring in a two-dimensional environment. However, the same skills can also be defined in a three-dimensional environment.

\subsubsection{Move-to-touch skill}

The move-to-touch skill is the transition motion in which a grasped object continues moving in a constant direction until it contacts another object (see Figure 3a). This skill includes sliding transition while maintaining touch in a different direction of movement. The skill is performed using a velocity control mode. The achievement of this skill can be judged by a sharp increase of resistance during the task. 


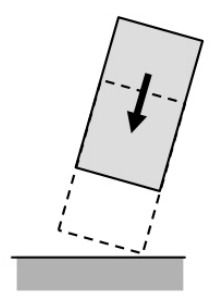

(a) Move-to-touch skill

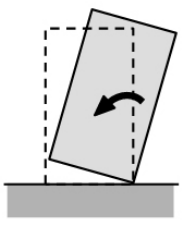

(b) Rotate-to-level skill

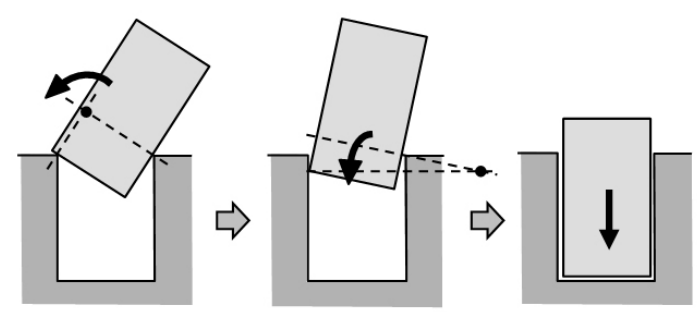

(c) Rotate-to-insert skill

Figure 3. Three important skill primitives

\subsubsection{Rotate-to-level skill}

The rotate-to-level skill is a rotary motion in which a grasped object continues revolving until it matches another object (see Figure 3b). The pushing force is maintained to keep the revolving object in contact with the other object while this skill is being performed. The attainment of the skill can be sensed by a slight moving of the instantaneous center.

\subsubsection{Rotate-to-insert skill}

The rotate-to-insert skill is a rotation of a grasped object slantingly into a dent in another object and the sequential transition while the grasped object is being pressed against the other object to achieve the goal (see Figure 3c). In a pegin-hole task, the grasped object can not easily be inserted directly into the hole since the clearance between the object and the hole is small in most cases. Therefore, the initial state in Figure $3 \mathrm{c}$ is derived by using other motions such as move-to-touch skills, and the centering state in Figure $3 \mathrm{c}$ is achieved by gradually raising the grasped object. The pushing force in the direction of the hole is maintained until the final state in Figure $3 \mathrm{c}$ is accomplished.

We will illustrate two other skills used in the loosening tasks of screws to be described later.

\subsubsection{Rotate-to-bite skill}

The rotate-to-bite skill is a rotation of a screwdriver which continues until a fit between the grooves of the screw head and the sharp point of the screwdriver succeeds (see Figure 4a). The pushing force is continued during this skill.

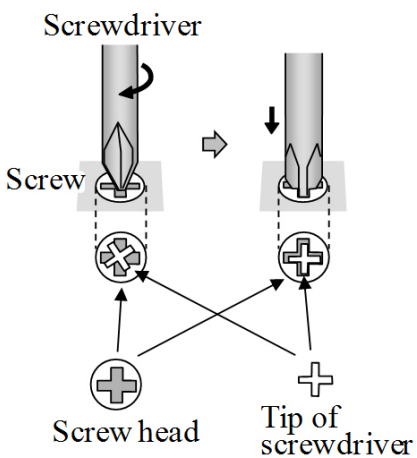

(a) Rotate-to-bite skill

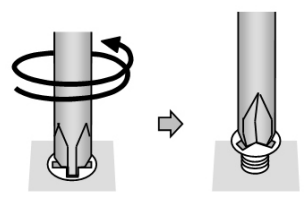

(b) Rotate-to-loosen skill
Figure 4. Two skill primitives in a screw loosening task using a screwdriver

\subsubsection{Rotate-to-loosen skill}

The rotate-to-loosen skill is a rotary motion of a screwdriver in order to loosen a screw (see Figure $4 \mathrm{~b}$ ). In this rotation motion, it is necessary to keep unifying the axes of rotation of the screw and the screwdriver. If the matching does not succeed, the screwdriver must be transferred to the correct position before the execution of the rotary motion begins. In this skill, the pushing force is continued, similarly to the above skills.

\subsection{Composition of skill sequence}

Most manipulation tasks consist of sequences of motion primitives such as move-to-touch, rotate-to-level and rotateto-insert skills. The sequences of the motion primitives can be derived by various methods. We have previously demonstrated a method which uses changes in the number of contact points in the skill primitive. ${ }^{[5]}$ Many other researchers have derived sequences of motion primitives by using geometric constraints $^{[19-21]}$ and Petri nets. ${ }^{[22,23]}$

We explained the derivation of skill primitives by decomposing manipulation tasks. On the other hand, when the task which should be performed was designated, it's almost possible to compose a task by connecting skill primitives. Programs of the fundamental skill primitives are always saved as software in the computer. If an execution task is appointed, the task is resolved into sequence of motion primitives, and programs of the basic skill primitives prepared in the computer are applied. If necessary, softwares of skill primitives must be made newly. The ordered task is composed of applied skill primitives, and the task is carried out. Therefore, it is important in practical use that many fundamental skill primitives which become common in various tasks are derived. Moreover, it is desirable that execution programs of most skill primitives are prepared in the computer software. 


\subsection{Process flow of skill-based manipulation}

To perform a manipulation task which consists of skill primitives, it is essential to clarify the processes of sensing, modeling, planning and execution. ${ }^{[8,9]}$ If accurate geometric models in the working environment have been constructed by visual sensing, the overall planning of the manipulator can be obtained only by the data of initial sensing. In reality, however, it is impossible to ignore data errors in visual sensing. Therefore, it is important to perform sensing and modeling just before performing the planning of a task and the skills constituting the task in order to restrain errors at each step. The procedures composed of visual sensing, geometric modeling, planning and execution are shown in Figure 5. In this scheme, the process flow consists of two layers: the task level and the skill level. First, the sensing, modeling and planning of the task level are carried out. Secondly, various components of the skill level are carried out, similarly.

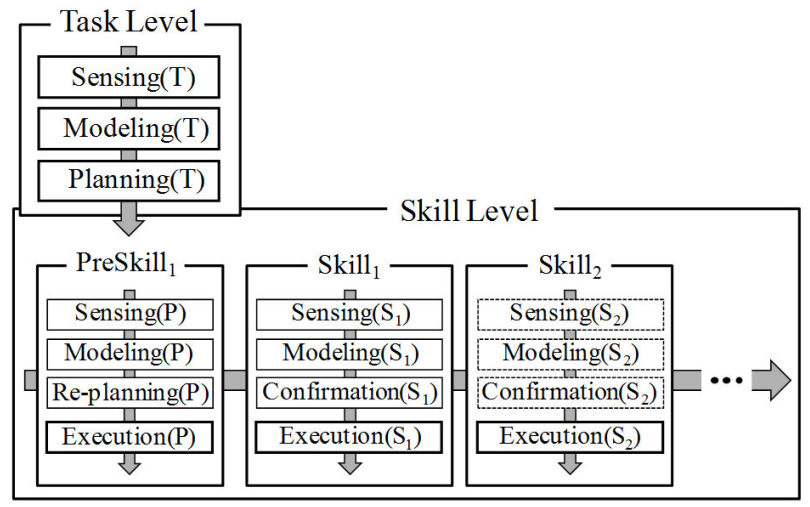

Figure 5. Process flow

\section{Step 1: Task level}

First, visual sensing is performed for the working environment of the manipulator and geometric modeling is done using the sensing data. Next, motion planning follows, and the sequences of the skill commands and the first position and orientation of the object clasped by the manipulator are decided.

The global object arrangement in the operating environment of the manipulator is fixed in the visual sensing and modeling of the task level. Some uncertainty often exists in the models of the working environment since the used data are global and rough. Therefore, there is the possibility that the planning may also have some uncertainty at the task level.

Step 2: Skill level

At the skill level, each motion primitive in the skill sequence is $\left\{\right.$ Skill $_{1}$, Skill $\left._{2}, \cdots\right\}$ which means the command sequence for the manipulator is performed in sequence. Before the

Published by Sciedu Press practice of this sequence, the gripped object is transitioned to the first state and this is indicated by PreSkill $_{1}$.

$\operatorname{PreSkill}_{1}\left(=\right.$ Skill $\left._{0}\right)$

First, visual sensing is performed for the object possessing the goal as near as possible from the course of the transition of the gripped object that was planned in $S_{k i l l}$. Next, geometric modeling and planning are carried out in succession. Planning can be done more accurately since the used data are local and precise. Therefore, the planning in PreSkill $_{1}$ is re-planning, and a more accurate start state can be derived. Then, the transference of the grasped object to the first state is performed.

Skill $_{i}\{i=1,2, \cdots\}$

At $\operatorname{Skill}_{i}\{i=1,2, \cdots\}$ in the skill level, the visual sensing, modeling and execution commands of each skill primitive for the manipulator are carried out. Next, the same processes are performed for each $\operatorname{Skill}_{i}\{i=2,3, \cdots\}$. After sensing and modeling are done, confirmation is performed. In this step, the initial state of execution of $\operatorname{Skill}_{i}\{i=1,2, \cdots\}$ is checked to confirm whether it corresponds to the scenario. The steps of sensing, modeling and confirmation in Skill $_{i}$ can be left out if the gripped object definitely exists in the first state of $\operatorname{Skill}_{i}\{i=2,3, \cdots\}$ when $\operatorname{Skill}_{i}$ is accomplished.

\section{CONCEPT OF SIMPLIFIED MODELS BASED ON SKILLS}

In this section, let us show the simplified models to be used in the step of planning and the simplified models to be used in the step of visual sensing.

By taking into account the concept of skills, compensation for margins of errors or failures both in modeling and in planning is possible. For example, in the move-to-touch skill, the margin of error occurring in the course of the transition can be canceled. Similarly, errors in the rotating direction can be minimized both in the rotate-to-level skill and in the rotate-to-insert skill. Furthermore, simplified geometric models can be derived by permitting the largest values of errors or margins in most skill motions.

It is not indispensable to form geometric models which perfectly agree with actual objects, both in the planning used for manipulator execution and in the visual sensing used for model matching. By composing geometric models only using the data required for sensing and planning, the process can be facilitated. We have shown how to simplify the processes of planning and visual sensing by using simplified models $(S M) .{ }^{[8,9]}$ The regulation of simplified models for visual sensing and for planning is explained in the following items. 


\subsection{Simplified models for planning}

First, we will explain simplified models for planning (SMP). The models are geometric models that consist only of the requisite and minimum data with respect to the form, position and orientation for practice of planning in skill-based manipulation.

\subsection{Simplified models for visual sensing and model matching}

Secondly, we will explain simplified models for visual sensing and model matching $(S M V)$. The models are geometric models that consist only of the required and minimum data for the form, position and orientation needed to execute model matching. SMP are derived from the several elements composing the simplified models for visual sensing and model matching $(S M V)$.

$S M P$ are geometric models including the restrictions on the trajectories of objects. For example, there are SMP which represent permissible trajectory errors to the shapes of objects. Furthermore, for planning in consideration of obstacle avoidance, there are $S M P$ including conditions not to contact other objects when moving. As for $S M V$, equivalence of the positions and orientations between the simplified model $S M V$ and the real object is the most important. The simplified models $S M V$ include the lines or the surfaces used to decide the positions and orientations. Therefore, it is necessary to be able to acquire the data of the lines or the surfaces from the real objects in visual sensing. Furthermore, a simplified model isn't always only one with respect to $S M P$ and $S M V$. More than one model may exist for a kind of the real object.

Simplified models $S M P, S M V$ can be obtained in each skill primitive $\operatorname{Skill}_{i}\{i=0,1,2, \cdots\}$. We will often express $S M P$ and $S M V$ in $S k i l l_{i}$ using $S M P_{i}$ and $S M V_{i}$, respectively.

\section{Simplified MODELS BASED ON SKILlS FOR INDUSTRIAL PRODUCTS}

For objects produced in factories, geometric models that are the same as the real objects are available in computer software. In this section, we will show SMP and $S M V$ when precise geometric models such as CAD models are available.

To make this explanation easier to understand, we show simplified models of screws in the task of loosening a screw using a screwdriver as examples. ${ }^{[8,9]}$ Although there are various types of screws and screwdrivers as shown in the left columns of Table $1,{ }^{[24]}$ we consider the screw-loosening task by uniting the following sequences of skills as shown in Figure 6 and Figure 7.

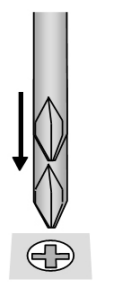

Skill $_{1}$

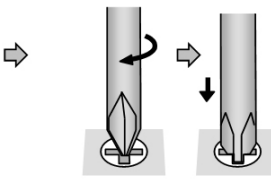

Skill $_{2}$

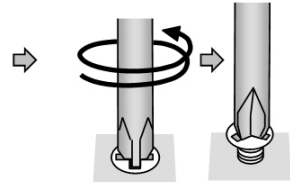

$\mathrm{Skill}_{3}$

Figure 6. Skill sequence of screw-loosening task using a cross-head screwdriver

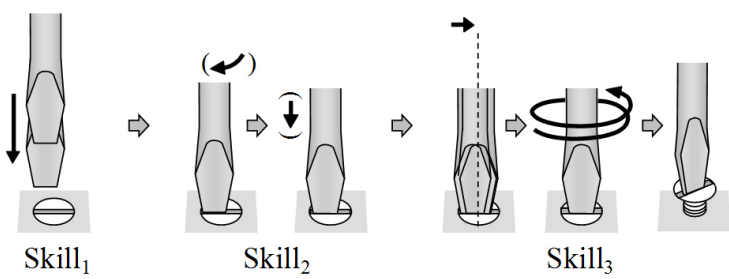

Figure 7. Skill sequence of loosening using a slotted screwdriver

Skill $l_{1}$ : Move-to-touch skill

$\mathrm{Skill}_{2}$ : Rotate-to-bite skill

$\mathrm{Skill}_{3}$ : Rotate-to-loosen skill

Next, we will show the simplified models of screws used in each skill primitive $\operatorname{Skill}_{i}\{i=0,1,2,3\}$, for several types of screws.

First, we consider the task for a crosshead screw (see Table 1, a and b) as shown in Figure 6. Skill ${ }_{1}$ is accomplished if the tip of the screwdriver enters a gutter near the center of the screw head. The simplified model for planning $S M P_{1}$ in $S k i l l_{1}$ is created according to the shape of the gutter (see Table 1, a and $b$ ). The shape is shown as a circle to exclude any dependency on the relative orientations of the screwdriver and the screw. The radius in a rare Reed and Prince screw which has little clearance (see Table 1b) is smaller than that in a typical Phillips screw (see Table 1a). The region allowed as the initial state of the move-to-touch skill of $S_{k i l l}$ is derived from a set of trajectories back-projected from the circle. The simplified model $S M V_{1}$ in $S k i l l_{1}$ is depicted by the outside circle of the screw head (see Table 1, a and b). The position and orientation of the circle of $S M P_{1}$ is derived from data of the circle of $S M V_{1}$ obtained by visual sensing. Simplified models $S M P_{i}, S M V_{i}$ in $\operatorname{Skill}_{i}\{i=2,3\}$ are not required since these skills are performed continuously (see Table 1, $\mathrm{a}$ and b). Additionally, simplified models $S M P_{0}, S M V_{0}$ in PreSkill $_{1}\left(\right.$ Skill $\left._{0}\right)$ are similar to the simplified models $S M P_{1}$, $S M V_{1}$ in $S k i l l_{1}$ since $S k i l l_{0}$ is the motion of transition to the initial region of $S_{k i l l}$. 
Table 1. Simplified models in screw-loosening task using a screwdriver

\begin{tabular}{|c|c|c|c|c|c|c|c|c|c|c|c|}
\hline \multirow{2}{*}{\multicolumn{2}{|c|}{ Type of screw }} & \multirow{2}{*}{ RO } & \multirow{2}{*}{$\begin{array}{l}\text { Type of } \\
\text { screwdriver } \\
\text { used }\end{array}$} & \multicolumn{2}{|c|}{ 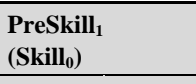 } & \multicolumn{2}{|l|}{ Skill $_{1}$} & \multicolumn{2}{|l|}{ Skill $_{2}$} & \multicolumn{2}{|l|}{$\mathrm{Skill}_{3}$} \\
\hline & & & & SMP $_{0}$ & SMV $_{0}$ & SMP $_{1}$ & $\mathrm{SMV}_{1}$ & SMP $_{2}$ & $\mathrm{SMV}_{2}$ & $\mathrm{SMP}_{3}$ & $\mathrm{SMV}_{3}$ \\
\hline \multirow{2}{*}{ I } & (a) Phillips & (3) & $\Leftarrow$ & 0 & & 0 & & $\begin{array}{l}\text { Not } \\
\text { needed }\end{array}$ & $\begin{array}{l}\text { Not } \\
\text { needed }\end{array}$ & $\begin{array}{l}\text { Not } \\
\text { needed }\end{array}$ & $\begin{array}{l}\text { Not } \\
\text { needed }\end{array}$ \\
\hline & (b) Reed and Prince & (5) & $\beta$ & 0 & 0 & 0 & & $\begin{array}{l}\text { Not } \\
\text { needed }\end{array}$ & $\begin{array}{l}\text { Not } \\
\text { needed }\end{array}$ & $\begin{array}{l}\text { Not } \\
\text { needed }\end{array}$ & $\begin{array}{l}\text { Not } \\
\text { needed }\end{array}$ \\
\hline II & (c) Slotted & (1) & $\square$ & (1]; & (1]; & (][) & (1]; & $\begin{array}{l}\text { Not } \\
\text { needed }\end{array}$ & $\begin{array}{l}\text { Not } \\
\text { needed }\end{array}$ & $\because$ & $\bigcirc$ \\
\hline \multirow{3}{*}{ III } & (d) Robertson & (D) & 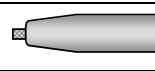 & 勧) & (a) & (a) & (a) & $\begin{array}{l}\text { Not } \\
\text { needed }\end{array}$ & $\begin{array}{l}\text { Not } \\
\text { needed }\end{array}$ & $\begin{array}{l}\text { Not } \\
\text { needed }\end{array}$ & $\begin{array}{l}\text { Not } \\
\text { needed }\end{array}$ \\
\hline & (e) Hex-drive & (1) & 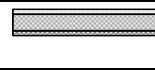 & ; & 余 & 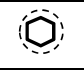 & 余 & $\begin{array}{l}\text { Not } \\
\text { needed }\end{array}$ & $\begin{array}{l}\text { Not } \\
\text { needed }\end{array}$ & $\begin{array}{l}\text { Not } \\
\text { needed }\end{array}$ & $\begin{array}{l}\text { Not } \\
\text { needed }\end{array}$ \\
\hline & (f) Two-Hole & 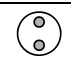 & & $\left(\begin{array}{l}0 \\
0 \\
0\end{array}\right.$ & $\left(\begin{array}{l}0 \\
0 \\
0\end{array}\right)$ & $\left(\begin{array}{c}0 \\
0 \\
0\end{array}\right)$ & (0) & $\begin{array}{l}\begin{array}{l}\text { Not } \\
\text { needed }\end{array} \\
\end{array}$ & $\begin{array}{l}\text { Not } \\
\text { needed }\end{array}$ & $\begin{array}{l}\text { Not } \\
\text { needed }\end{array}$ & $\begin{array}{l}\text { Not } \\
\text { needed }\end{array}$ \\
\hline \multirow{2}{*}{ IV } & \multirow{2}{*}{ (g) Cross \& slotted } & \multirow{2}{*}{ (5) } & & 0 & 0 & 0 & 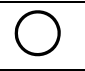 & $\begin{array}{l}\text { Not } \\
\text { needed }\end{array}$ & $\begin{array}{l}\text { Not } \\
\text { needed }\end{array}$ & $\begin{array}{l}\text { Not } \\
\text { needed }\end{array}$ & $\begin{array}{l}\text { Not } \\
\text { needed }\end{array}$ \\
\hline & & & ${ }^{\text {or }}$ & (1]; & \begin{tabular}{c}
11 \\
$J_{1}$ \\
\hdashline
\end{tabular} & (]) & $\left(\begin{array}{l}11 \\
\mathrm{JL}_{1}\end{array}\right.$ & $\begin{array}{l}\text { Not } \\
\text { needed }\end{array}$ & $\begin{array}{l}\text { Not } \\
\text { needed }\end{array}$ & $\because$ & 0 \\
\hline
\end{tabular}

Note. RO: Real objects; $\mathrm{SMP}_{\mathrm{i}}$ : Simplified models for planning in $\mathrm{Skill}_{\mathrm{i}}$; $\mathrm{SMV}_{\mathrm{i}}$ : Simplified models for visual sensing and model matching in Skill ${ }_{\mathrm{i}}$.

Furthermore, we consider a slotted screw, a Robertson screw, a hex-drive screw, a spanner screw, and a cross and slotted screw. Simplified models $S M P_{i}$ and $S M V_{i}$ can be derived as shown in Table 1 for each type of screw. Details are available in the references. ${ }^{[8,9]}$

\section{Simplified MODELS BASED ON SKILLS FOR OBJECTS USED IN DAILY LIFES}

For products made in factories, CAD models exist in most cases. Therefore the robots working in factories can perform their tasks with high reliability. Using simplification models facilitates the processes of sensing, modeling and planning for the execution of tasks, and simplified models $S M P_{i}$ and $S M V_{i}$ are derived for each object and for each skill primitive comprising the tasks.

However, unlike factory products, few precise models such as CAD models are presently available for objects used in daily life. For robots working in human daily life environments, general models used in general object recognition can be applied to sensing, modeling and planning in many cases. When a general model closely resembles a real object, highly reliable tasks become possible by using the ability of the manipulation skills to cancel errors. Conversely, when the difference between the real object and general model is large, the operational reliability is lost, and the task often fails.

\subsection{Process of a task for daily life}

The reliability of the achievement of a task greatly varies depending on the difference in the size of the real object and general model as mentioned above. In this section, we consider the process of a task by classifying it in two cases Published by Sciedu Press by the difference in size.

\section{Case 1}

This is a case in which the size difference is zero or small. In this case, highly precise geometric models can be constructed since the shape of the real object has been obtained correctly.

The ordered tasks can be performed similarly by using simplified models based on skills for industrial products as mentioned in Section 4 in each section of sensing, modeling and planning.

\section{Case 2}

This is a case in which the size difference is relatively large. In this case, it is difficult to construct precise geometric models since there are not enough data to create an exact model.

The reliability of the achievement of the task is low when planning and execution are performed directly using models generated only using known data. In this case, two steps of planning are performed as follows.

(1) First, planning is performed for general models often used in object recognition. This part is the same as the planning section in Case 1.

(2) Second, planning for extended models for general models is performed. This planning is performed for a large extended size to increase reliability.

\subsection{Simplified models of objects used in daily life}

Here we consider a plastic bottle and a cup as objects used in daily life. Both objects are now commonplace in human life. We will consider the tasks involved in repacking a plastic bottle from a large box into a small box, and the tasks involved 
in transferring a cup from a cupboard to a table. In these examples, we assume that there are no precise models such as CAD models available in computer software for these plastic bottles and cups. Therefore, the procedure in Case 2 in Section 5.1 is taken into account.

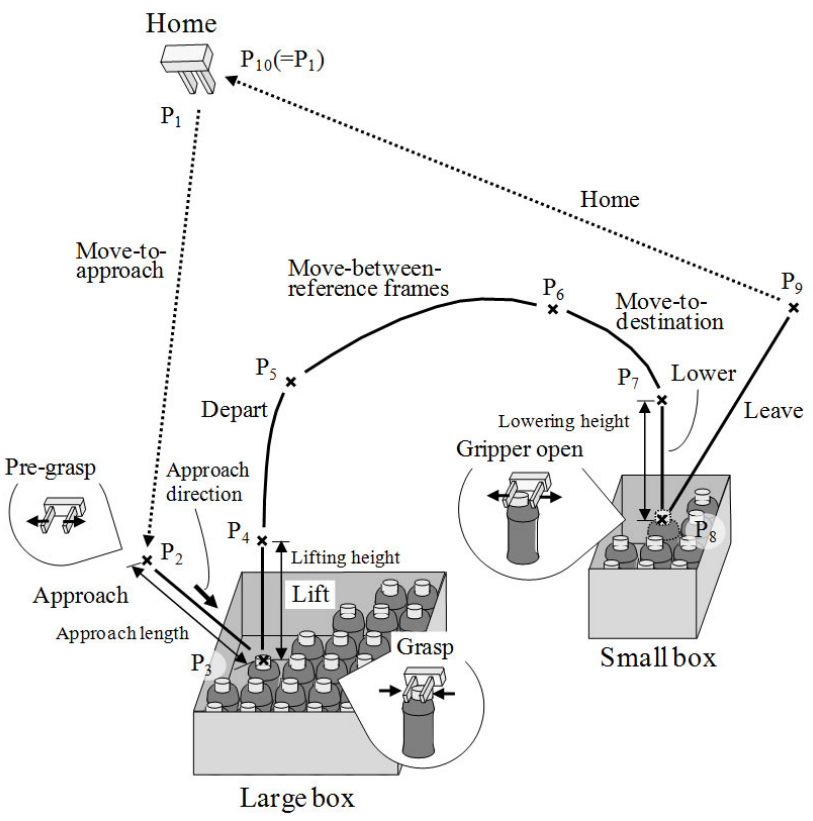

Figure 8. Pick-and-place task using a gripper for plastic bottles

\subsubsection{Simplified models in the transfer task for plastic bot- tles}

This task involves the transfer of plastic bottles in which the bottles are picked and placed using a gripper as shown in Figure 8. Figure 9 shows command sequences of the transfer and manipulation tasks composed of 12 skill primitives. A plastic bottle containing a beverage is repacked from a large case into a small case through the execution of this task. It is assumed that cross sections of the cap and the body of a real bottle consist of n-polygonal $(n($ cap $)=10, n($ body $)=10$ in Table 2a).

First, simplified models $S M P_{i}, S M V_{i}$ of the plastic bottle in step (1) in Case 2 are derived as shown in Table 2a. $M(k)$ and $m(k)(k=I I, I I I, I V, V)$ in the $S M P_{i}$ are the radii of a circumscribed circle and inscribed circle, respectively. Twelve skill primitives are summarized in six sections I to VI to facilitate planning by using the same models as shown in Table 2a. In sections II and $\mathrm{V}$, the radii $M(k)$ and $m(k)(k=I I, V)$ of the model for the cap are the indexes used for the opening width between the fingers of the gripper when grasping and opening. The opening width between the fingers is controlled so that the value of the width becomes larger than $M(k)$ at $S_{k i l l}$ and $S_{k i l l}{ }_{10}$ and becomes smaller than $m(k)$ at $S_{k i l l}$. In sections III and IV, the radius $M(k)(k=I I I, I V)$ of the model for the body of the bottle is the index used to derive the path of the transfer, check for obstacle avoidance, etc. The simplified models $S M V_{i}$ are drawn by a circle whose radius is $r_{v}(k)=(n(l) / \pi) \ln \{\tan (\pi /(2 n(l))+\pi / 4)\} m(k)(k=I I$, $I I I, I V, V)(l=$ cap, boby $)$, which is derived by the LMS technique. ${ }^{[9]}$

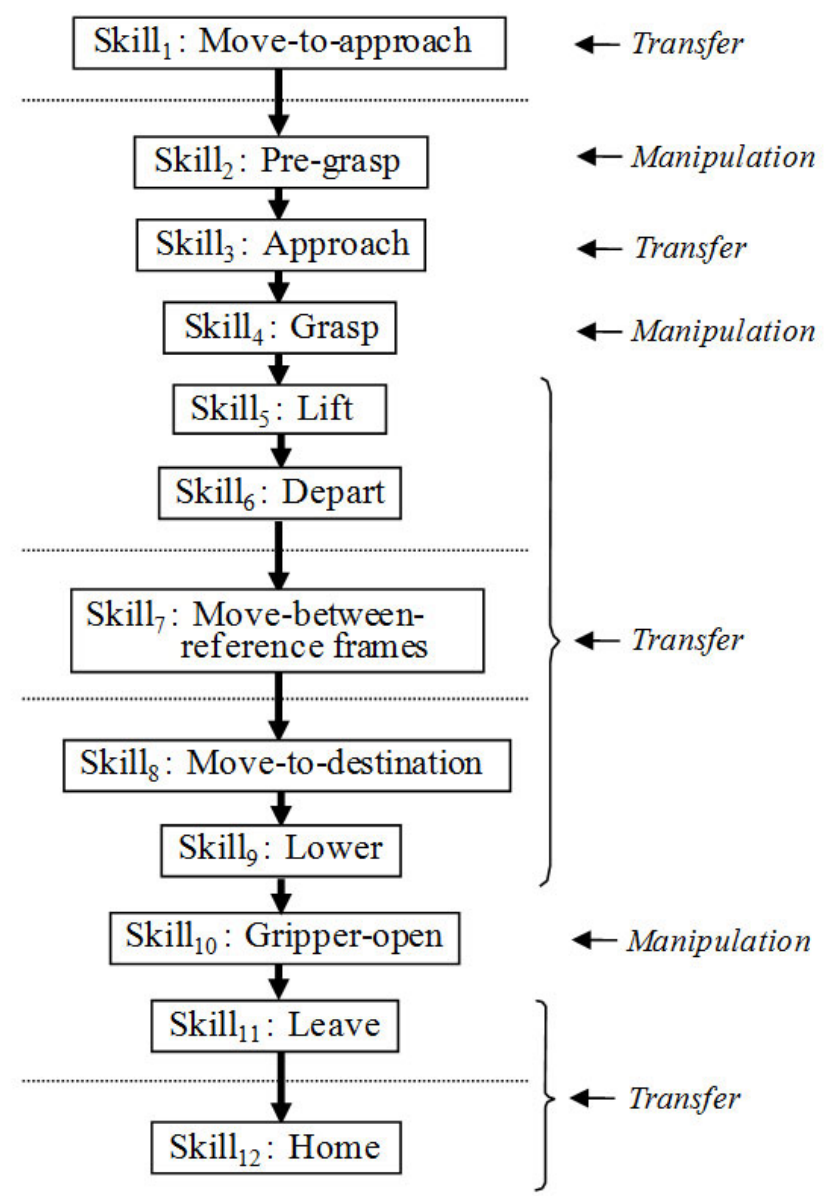

Figure 9. Command sequence of task

Secondly, simplified models $S M P_{i}, S M V_{i}$ of the plastic bottle in step (2) in Case 2 are derived as shown in Table $2 b$. $M^{\prime}(k)$ and $m^{\prime}(k)(k=I I, I I I, I V, V)$ are the radii of the circles where $M^{\prime}(k)>M(k)>m(k)>m^{\prime}(k)$ in sections II, III, IV and $\mathrm{V}$. The values of $M^{\prime}(k)$ and $m^{\prime}(k)$ are decided as values that can avoid the failure of the task. The fingers of the gripper are controlled so that the opening width between the fingers becomes larger than the value of $M^{\prime}(k)$ at $S_{k i l l}$ and $S_{k i l l}{ }_{10}$ and becomes smaller than the value of $m^{\prime}(k)$ at $S_{k i l l_{4}}$. 
Table 2. Simplified models of plastic bottles in pick-and-place task using a gripper

(a) With use of a CAD model

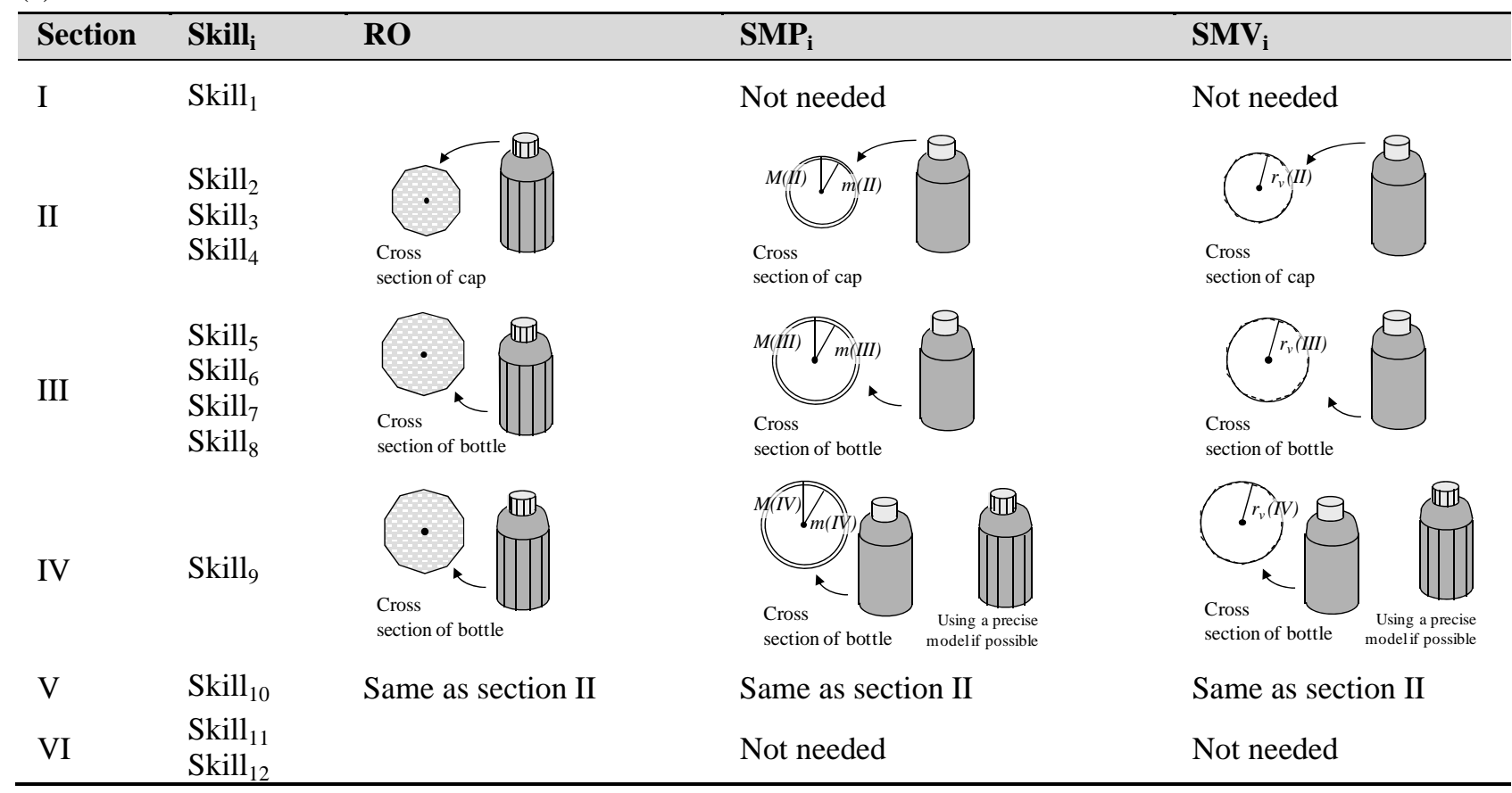

(b) Without use of a CAD model

\begin{tabular}{|c|c|c|c|c|}
\hline Section & Skill $_{i}$ & RO & $\mathbf{S M P}_{i}$ & $\mathbf{S M V}_{\mathbf{i}}$ \\
\hline I & Skill $_{1}$ & & Not needed & Not needed \\
\hline II & $\begin{array}{l}\text { Skill }_{2} \\
\text { Skill }_{3} \\
\text { Skill }_{4}\end{array}$ & $\begin{array}{l}\text { Cross } \\
\text { section of cap }\end{array}$ & Cross & $\begin{array}{l}\text { Cross } \\
\text { section of cap }\end{array}$ \\
\hline III & $\begin{array}{l}\text { Skill }_{5} \\
\text { Skill }_{6} \\
\text { Skill }_{7} \\
\text { Skill }_{8}\end{array}$ & $\begin{array}{l}\text { Cross } \\
\text { section of bottle }\end{array}$ & 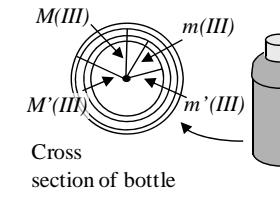 & Cross \\
\hline IV & Skill $_{9}$ & $\begin{array}{l}\text { Cross } \\
\text { section of bottle }\end{array}$ & $\begin{array}{l}\text { Cross } \\
\text { section of bottle }\end{array}$ & $\begin{array}{l}\text { Cross } \\
\text { section of bottle }\end{array}$ \\
\hline V & Skill $_{10}$ & Same as section II & Same as section II & Same as section II \\
\hline VI & $\begin{array}{l}\text { Skill }_{11} \\
\text { Skill }_{12}\end{array}$ & & Not needed & Not needed \\
\hline
\end{tabular}

Note. RO: Real objects; $\mathrm{SMP}_{\mathrm{i}}$ : Simplified models for planning in Skill $\mathrm{i}_{\mathrm{i}} \mathrm{SMV}_{\mathrm{i}}$ : Simplified models for visual sensing and model matching in Skill $\mathrm{l}_{\mathrm{i}}$

Although sections III and IV are performed in succession, the reason for dividing these sections is to restrain flexibility and treat the object precisely in the lowering skill of section IV.

Simplified models are derived for only the necessary parts in the objects in each section, and highly reliable task execuPublished by Sciedu Press tion becomes possible by performing sensing, modeling and planning for the simplified models.

\subsubsection{Simplified models in the transfer task for a cup}

This task involves the transfer of a cup such as a coffee cup or teacup in which the cup is picked and placed using a gripper as shown in Figure 10. The command sequences are the 
same as in Figure 9 which is composed of 12 skill primitives. cution of this task. It is assumed that the cross section of a A cup is moved from a cupboard to a table through the exe- real cup is an ellipse approximating a circle.

Table 3. Simplified models of cups in pick-and-place task using a gripper

(a) With use of a CAD model

\begin{tabular}{|c|c|c|c|c|}
\hline Section & Skill $_{i}$ & RO & $\mathbf{S M P}_{\mathbf{i}}$ & $\mathrm{SMV}_{\mathbf{i}}$ \\
\hline I & Skill $_{1}$ & & Not needed & Not needed \\
\hline II & $\begin{array}{l}\text { Skill }_{2} \\
\text { Skill }_{3} \\
\text { Skill }_{4}\end{array}$ & $\begin{array}{l}\text { Cross } \\
\text { section of cup }\end{array}$ & $\begin{array}{l}\text { Cross } \\
\text { section of cup }\end{array}$ & $\begin{array}{l}\text { Cross } \\
\text { section of cup }\end{array}$ \\
\hline III & $\begin{array}{l}\text { Skill }_{5} \\
\text { Skill }_{6} \\
\text { Skill }_{7} \\
\text { Skill }_{8} \\
\text { Skill }_{9}\end{array}$ & $\begin{array}{l}\text { Cross } \\
\text { section of cup }\end{array}$ & $\begin{array}{l}\text { Cross } \\
\text { section of cup }\end{array}$ & $\begin{array}{l}\text { Cross } \\
\text { section of cup }\end{array}$ \\
\hline IV & Skill $_{10}$ & Same as section II & Same as section II & Same as section II \\
\hline $\mathrm{V}$ & $\begin{array}{l}\text { Skill }_{11} \\
\text { Skill }_{12}\end{array}$ & & Not needed & Not needed \\
\hline
\end{tabular}

(b) Without use of a CAD model

\begin{tabular}{|c|c|c|c|c|}
\hline Section & Skill $_{i}$ & RO & $\mathrm{SMP}_{\mathbf{i}}$ & $\mathrm{SMV}_{\mathbf{i}}$ \\
\hline $\mathrm{I}$ & Skill $_{1}$ & & Not needed & Not needed \\
\hline II & $\begin{array}{l}\text { Skill }_{2} \\
\text { Skill }_{3} \\
\text { Skill }_{4}\end{array}$ & $\begin{array}{l}\text { Cross } \\
\text { section of cup }\end{array}$ & $\begin{array}{l}\text { Cross } \\
\text { section of cup }\end{array}$ & $\begin{array}{l}\text { Cross } \\
\text { section of cup }\end{array}$ \\
\hline III & $\begin{array}{l}\text { Skill }_{5} \\
\text { Skill }_{6} \\
\text { Skill }_{7} \\
\text { Skill }_{8} \\
\text { Skill }_{9}\end{array}$ & $\begin{array}{l}\text { Cross } \\
\text { section of cup }\end{array}$ & $\begin{array}{l}\text { Cross } \\
\text { section of cup }\end{array}$ & $\begin{array}{l}\text { Cross } \\
\text { section of cup }\end{array}$ \\
\hline IV & Skill $_{10}$ & Same as section II & Same as section II & Same as section II \\
\hline V & $\begin{array}{l}\text { Skill }_{11} \\
\text { Skill }_{12}\end{array}$ & & Not needed & Not needed \\
\hline
\end{tabular}

Note. RO: Real objects; $\mathrm{SMP}_{\mathrm{i}}$ : Simplified models for planning in $\mathrm{Skill}_{\mathrm{i}}$; $\mathrm{SMV}_{\mathrm{i}}$ : Simplified models for visual sensing and model matching in Skill $\mathrm{l}_{\mathrm{i}}$.

First, simplified models $S M P_{i}, S M V_{i}$ of the cup in step (1) in Case 2 are derived as shown in Table 3a. $M(k)$ and $m(k)(k$ $=I I, I I I, I V)$ in the $S M P_{i}$ are the radii of the circumscribed circle and inscribed circle of the ellipse, respectively. Twelve skill primitives are summarized in sections I to $\mathrm{V}$ to facilitate the planning. Five skills $S_{k i l l}$ to $S_{k i l l}$ are summarized in one section since it is not necessary to place the cup in a narrow space in $S_{k i l l}$, unlike that in Section 5.2.1. In sections 
II and IV, the radii $M(k)$ and $m(k)(k=I I, I V)$ of the model for the cup are the indexes used for the opening width between the fingers of the gripper. In section III, the radius $M$ of the model for the cup is the index used for planning the transfer in order to avoid obstacles.

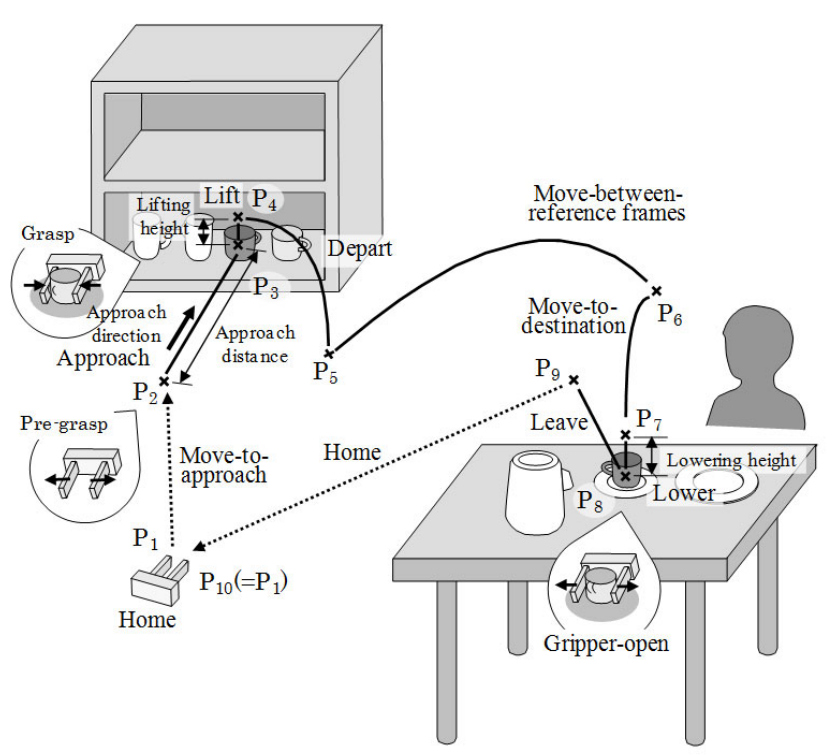

Figure 10. Pick-and-place task using a gripper for cups

Secondly, simplified models $S M P_{i}, S M V_{i}$ of the cup in step (2) in Case 2 are derived as shown in Table 3b. $M^{\prime}(k)$ and $m^{\prime}(k)(k=I I, I I I, I V)$ are the radii of circles where $M^{\prime}(k)$ $>M(k)>m(k)>m^{\prime}(k)$ in sections II and IV. The values of $M^{\prime}(k)$ and $m^{\prime}(k)$ are decided as values that can avoid the failure of the task. The opening width between the fingers of the gripper should be larger than the value of $M^{\prime}(k)$ at $S k i l l_{2}$ and $S_{k i l l} l_{10}$ and should be smaller than the value of $m^{\prime}(k)$ at Skill $_{4}$.

When approaching $\left(\mathrm{Skill}_{3}\right)$ and grasping a cup $\left(\mathrm{Skill}_{4}\right)$ are performed, the cup handle often becomes an obstacle. For simplification, we do not take into account the cup handle in this paper. If the cup handle were to be considered as an obstacle, additional tasks such as rotating the cup or transferring another cup often occur.

Simplified models for the two tasks are shown in Sections 5.2.1 and 5.2.2. Task planning using manipulation skills has the ability to cancel an error occurring from modeling errors. Reliable planning can be performed by using skill-derived simplified models. However, still there is a possibility that an error might occur. When an error occurs, a return operation should be performed using the error recovery techniques we have studied. ${ }^{[25,26]}$

\section{Conclusions}

We have described a method to derive highly reliable planning of tasks by using manipulation skill-based simplified models for objects used in the daily life of humans. While there are precise models for products made at factories in many cases, precise models for objects used in daily life are not always available in computer software. In such cases, failures often occur since models in the working environment become indistinct. Nevertheless, it is necessary to perform ordered tasks with high reliability. The derivation method of simplified models based on skills for objects used in daily lifes which we proposed greatly contributes to it.

We have been researching error recovery techniques. ${ }^{[25,26]}$ It is important to apply error recovery techniques for manipulation tasks in daily life to increase their reliability. Moreover, it is essential to achieve stable, error-free manipulation tasks for high reliability. In this paper, simplified models derived for objects produced in factories were applied to objects used in daily life. These simplified models were obtained by extending general models used in object recognition. Sensing, modeling and planning are performed based on these simplified models.

In the future, we will further study how to derive skill-based simplified models for many objects used in daily life and how to summarize skill sequences into several sections to execute tasks effectively. It is also important to execute tasks with high reliability in crowded environments and when obstacles exist. In this paper, we showed only geometric models definition about simplified geometric models in skill-based manipulation for objects used in daily life. In the future, it is necessary to apply our technique to a real robot system or the simulator.

\section{ACKNOWLEDGEMENTS}

This work was supported by JSPS KAKENHI Grant Number $15 \mathrm{~K} 00370$.

\section{REFERENCES}

[1] Hasegawa T, Suehiro T, Ogasawara T, et al. An integrated telerobotics system with a geometric environment model and manipulation skills. Proc. IEEE Int. Workshop on Intell. Robots Syst.
Tsuchiura, Japan. 1990: 335-41. https://doi.org/10.1109/IR OS. 1990.262400

[2] Hasegawa T, Suehiro T, Takase K. A robot system for unstructured environments based on an environment model and manipulation 
skills. Proc. IEEE Int. Conf. Robot. Autom. Sacramento, USA. 1991: 916-23. https://doi.org/10.1109/ROBOT.1991.131705

[3] Hasegawa T, Suehiro T, Takase K. A model-based manipulation system with skill-based execution in unstructured environment. Proc. Int. Conf. on Advanced Robotics. Pisa, Italy. 1991: 970-5. https://doi.org/10.1109/ICAR. 1991.240546

[4] Hasegawa T, Suehiro T, Takase K. A model-based manipulation system with skill-based execution. IEEE Trans. Robot. Autom. 1992; 8(5): 535-44. https : //doi.org/10.1109/70.163779

[5] Nakamura A, Ogasawara T, Suehiro T, et al. Skill-based backprojection for fine motion planning. Proc. IEEE/RSJ Int. Conf. on Intell. Robots Syst. Osaka, Japan. 1996; 2: 526-33. https : //doi.org/10.1109/IROS.1996.570846

[6] Nakamura A, Ogasawara T, Suehiro T, et al. Fine motion strategy in three-dimensional space using skill-based backprojection. Artificial Life and Robotics. Springer. 1998; 2(3): 134-7. https : //doi.org/10.1007/BF02471170

[7] Nakamura A, Suehiro T, Machida K, et al. Fine Motion Planning Using Skill-Based Backprojection to Handle Control and Visual Errors. Proc. Int. Con. on Advanced Robotics. Tokyo, Japan. 1999: 31-8.

[8] Nakamura A, Ogasawara T, Kitagaki K, et al. Using robust and simplified geometric models in skill-based manipulation. Proc. IEEE/RSJ Int. Conf. on Intell. Robots Syst. Hawaii, USA. 2001; 1: 139-45. https://doi.org/10.1109/IROS. 2001.973349

[9] Nakamura A, Kitagaki K, Suehiro T. Using Simplified Geometric Models in Skill-Based Manipulation. Advanced Robotics. 2004; 18(8): 835-58. https : //doi.org/10.1163/1568553041738130

[10] Ricker SL, Sarkar N, Rudie K. A Discrete-Event Systems Approach to Modeling Dextrous Manipulation. Robotica. 1996; 14: 515-25. https://doi.org/10.1017/S0263574700020002

[11] McCarragher BJ, Asada H. A discrete Event Approach to the Control of Robotic Assembly Tasks. Proc. IEEE Int. Conf. Robot. Autom. USA. 1993: 331-6. https://doi.org/10.1109/ROBOT.1993.2 92003

[12] Ostrovsky-Berman Y, Joskowicz L. Geometric Computation for Assembly Planning with Planar Toleranced Parts. Proc. IEEE Int. Conf. Robot. Autom. Barcelona, Spain. 2005: 4103-10. https: //doi.org/10.1109/ROBOT.2005.1570748

[13] Kent D, Chernova S. Construction of an Object Manipulation Database from Grasp Demonstrations. Proc. IEEE/RSJ Int. Conf. on Intell. Robots Syst. Chicago, USA. 2014: 3347-52. https : //doi.org/10.1109/IROS.2014.6943028

[14] Paolini R, Mason MT. Data-Driven Statistical Modeling of a Cube Regrasp. Proc. IEEE/RSJ Int. Conf. on Intell. Robots Syst. Daejeon,
Korea. 2016: 2554-60. https ://doi .org/10.1109/IROS. 2016. 7759397

[15] Kriegel S, Brucker M, Marton ZC, et al. Combining Object Modeling and Recognition for Active Scene Exploration. Proc. IEEE/RSJ Int. Conf. on Intell. Robots Syst. Tokyo, Japan. 2013: 2384-91. https://doi.org/10.1109/IROS. 2013.6696691

[16] Wagner R, Frese U, Bauml B. 3D Modeling, Distance and Gradient Computation for Motion Planning: A Direct GPGPU Approach. Proc. IEEE Int. Conf. Robot. Autom. Karlsruhe, Germany. 2013: 3571-7.

[17] Meißner P, Hanselmann F, Jakel R, et al. Automated Selection of Spatial Object Relations for Modeling and Recognizing Indoor Scenes with Hierarchical Implicit Shape Models. Proc. IEEE/RSJ Int. Conf. on Intell. Robots Syst. Hamburg, Germany. 2015: 4257-64.

[18] Li B, Chen H, Jin T. Industrial Robotic Assembly Process Modeling Using Support Vector Regression. Proc. IEEE/RSJ Int. Conf. on Intell. Robots Syst. Chicago, USA. 2014: 4334-9. https : //doi .or g/10.1109/IROS. 2014.6943175

[19] Xiao J, You S. Haptic Modeling Based on Contact and Motion Types. Proc. IEEE/RSJ Int. Conf. on Intell. Robots Syst. Lausanne, Switzerland. 2002: 2925-30.

[20] Pan F, Schimmels JM. Efficient Contact State Graph Generation for Assembly Applications. Proc. IEEE Int. Conf. Robot. Autom. Taipei, Taiwan. 2003: 2592-8.

[21] Aleotti J, Caselli S. Efficient Planning of Disassembly Sequences in Physics-Based Animation. Proc. IEEE/RSJ Int. Conf. on Intell. Robots Syst. St. Louis, USA. 2009: 87-92. https ://doi .org/10 .1109/IROS. 2009.5354486

[22] Huang YS, Xie X, Hsu CN. Modeling and Analysis Manufacturing Systems Using a New Class of Petri Nets. Proc. IEEE Int. Conf. Robot. Autom. New Orleans, USA. 2004: 554-9.

[23] Costelha H, Lima P. Modelling, Analysis and Execution of Robotic Tasks using Petri Nets. Proc. IEEE/RSJ Int. Conf. on Intell. Robots Syst. San Diego, USA. 2007: 1449-54.

[24] Jackson A, Day D. Tools and How to Use Them. Wings Books. 1992: 220-6. PMid:1739796.

[25] Nakamura A, Kotoku T. Systematization of Error Recovery in Skill-Based Manipulation. Artificial Life and Robotics. Springer. 2009; 14(2): 203-8. https://doi.org/10.1007/s10015-009 $-0654-5$

[26] Nakamura A, Nagata K, Harada K, et al. Error Recovery Using Task Stratification and Error Classification for Manipulation Robots in Various Fields. Proc. IEEE/RSJ Int. Conf. on Intell. Robots Syst. Tokyo, Japan. 2013: 3535-42. https ://doi .org/10.1109/IROS .2013 .6696860 